\title{
Comparison of Light-Emitting Diode Fluorescent Microscopy Against Bright-Field Microscopy and JD-TB-Antigen Test for the Diagnosis of Pulmonary Tuberculosis
}

\author{
Boja Dufera Taddese ${ }^{1}$, Abraham Tesfaye Bika ${ }^{1,2}$, Abay Sisay Misganaw ${ }^{3,}$, , Silvia Blanco Palencia ${ }^{2}$, \\ Chala Chaburte Galata ${ }^{4}$, Abiy Aklilu Teferra ${ }^{5}$ \\ ${ }^{1}$ Addis Ababa Health Research and Laboratory, Addis Ababa, Ethiopia \\ ${ }^{2}$ College of Natural Sciences, Addis Ababa University, Addis Ababa, Ethiopia \\ ${ }^{3}$ Columbia University's Mailman School of Public Health, International Center for AIDS Care and Treatment Program(ICAP), Addis Ababa, \\ Ethiopia \\ ${ }^{4}$ Faculty of Veterinary Medicine, Addis Ababa University, Bishoftu, Ethiopia \\ ${ }^{5}$ Ethiopian Conformity Assessment Enterprise, Addis Ababa, Ethiopia
}

\section{Email address:}

duferataddese@yahoo.com (B. D. Taddese), atabrish@gmail.com (A. T. Bika), abusis27@gmail.com (A. S. Misganaw) sblanc1976@gmail.com (S. B. Palencia), chala892@yahoo.com (C. C. Galata), abiye24@yahoo.com (A. A. Teferra)

${ }^{*}$ Corresponding author

\section{To cite this article:}

Boja Dufera Taddese, Abraham Tesfaye Bika, Abay Sisay Misganaw, Silvia Blanco Palencia, Chala Chaburte Galata, Abiy Aklilu Teferra. Comparison of Light-Emitting Diode Fluorescent Microscopy Against Bright-Field Microscopy and JD-TB-Antigen Test for the Diagnosis of Pulmonary Tuberculosis. American Journal of Internal Medicine. Vol. 5, No. 5, 2017, pp. 105-111. doi: 10.11648/j.ajim.20170505.18

Received: November 30, 2016; Accepted: January 18, 2017; Published: October 26, 2017

\begin{abstract}
Bright field microscopy using Ziehl-Neelsen $(\mathrm{ZN})$ stained smear has been the major diagnostic technique for the diagnosis of pulmonary tuberculosis and treatment initiation in resource limited settings. However, it has its own limitation in terms of its sensitivity. Various reports have shown superior sensitivity of light emitting-diode fluorescent microscopy (LEDFM) and comparable specificity to bright field microscopy. The aim of the study was to compare the results of Auramine Ostained sputum smears by LED-FM against Ziehl-Neelsen stained sputum smears and JD-TB-Antigen test using TB culture as a reference test. A cross-sectional study was conducted in Southern Nations and Nationality People Regions (SNNPR) of Ethiopia from September 2013 to November 2014. A total of 248 sputum samples were collected from different Health Centers and Hospitals of the study area, analyzed by LED-FM and ZN stained bright field microscopy. The sensitivity, specificity, positive and negative predictive value were $66.12 \%, 95.28 \%, 93.02 \%$ and $74.69 \%$ for $\mathrm{ZN}$ stained microscopy; $81.82 \%$, $93.70 \%, 92.50 \%$ and $98.35 \%$ for LED-FM, and 58.33\%, 63.27\%, 53.85\% and $67.39 \%$ for JD-TB-Antigen test, respectively. The correlation between LED-FM, ZN and culture have shown statistically significant $(\mathrm{p}<0.001)$. The sensitivity of LED-FM is better than $\mathrm{ZN}$-stained bright field microscopy. It is also easy to perform, save time and better choice for sputum microscopic examination. The performance of JD-TB-Antigen test was very low for the diagnosis of tuberculosis in sputum specimens. Therefore, further study should be done to use JD-TB-Ag test for diagnosis of pulmonary tuberculosis.
\end{abstract}

Keywords: Ziehl-Neelsen, Auramine-O Stain, LED-FM, TB Culture

\section{Introduction}

Tuberculosis is an infectious disease caused mainly by bacillus. More than a century, despite directly observed treatment short course (DOTS) implementation in different countries over the world, tuberculosis remains one of the major causes of global death and constitutes a serious public health problem worldwide [1]. It ranks the second leading causes of death worldwide next to HIV/AIDS. The situation 
is aggravated in areas where HIV/AIDS is highly endemic especially in sub Saharan Africa including Ethiopia [2].

In Ethiopia, tuberculosis has been recognized as major public health problem in early 1950s. Efforts to control tuberculosis began in the early 1960 s with the establishment of TB centers and sanatoriums in major urban areas in the country. Ethiopia ranks seventh out of the world's 22 highburden countries (HBCs) for tuberculosis (TB). The emergence of Multidrug-Resistant (MDR)-TB is another challenge faced by the country. Ethiopia ranks fifteenth among the 27 multi-drug resistant TB (MDR-TB) priority countries over the world [3].

In developing countries, due to lack of appropriate, affordable and alternative diagnostic techniques, the control of the tuberculosis is lagged behind. ZN-stained sputum microscopy has been the most widely used diagnostic method. However, currently available diagnostic technologies including smear microscopy and culture have their own shortcoming and most clinical laboratories with the greatest test capacity and more accurate test methods are located in urban settings [4].

The most sensitive and current optimal method for the detection of active Mycobacterium tuberculosis Complex (MTBC) disease is via mycobacterium culture and it remains necessary for the definitive diagnosis of pulmonary TB in patients whose smear produces a negative result [5].

Fluorescent microscopy (FM) for the detection of tuberculosis has been in use since the mid-1940s and is the method of choice for large laboratories in industrialized nations [6]. A review have shown, FM on average $10 \%$ more sensitive than conventional light microscopy, have comparable specificity and takes significantly less time to read smears [7]. However, the cost and difficulties of its maintenance limits its implementation [8].

Even though bright field microscopy using $\mathrm{ZN}$ staining techniques is most commonly used diagnostic techniques for diagnosis of pulmonary tuberculosis, the diagnostic sensitivity of $\mathrm{ZN}$ is low especially in areas where overlapping synergy of HIV-TB is high. Various reports have shown superior sensitivity of LED-FM and comparable specificity compared to bright field microscopy. The application of rapid immunechromatographic antigen test hasn't been evaluated in different settings of developing countries.

This entails the need to oversee diagnostic technologies with lower cost, user friendly and better sensitivity and specificity. Hence this study was aimed to compare the performances of light-emitting-diode fluorescent microscopy against bright-field microscopy and JD-TB-Antigen test for the diagnosis of pulmonary tuberculosis using TB culture as reference test.

\section{Research Method and Design}

\subsection{Study Setting}

A Cross-sectional study was conducted in Southern Nations and Nationalities People Region of Ethiopia
(SNNPR) from November 2013 to September 2014. Microscopic and JD-Antigen tests were done in Addis Ababa Health Research Laboratory and culture was done in Armauer Hansen research institute (AHRI).

\subsection{Inclusion/Exclusion Criteria}

Pulmonary tuberculosis presumptive patients greater than or equal to 18 years who had visited the selected health centers and hospitals found in SNNPR of Ethiopia from November 2013 to September 2014 medical care were included in this study, whereas the rest were not included in this study.

\subsection{Study Participants and Ethics}

The study participants were pulmonary tuberculosis presumptive patients greater than or equal to 18 years who had visited the selected health centers and hospitals found in SNNPR of Ethiopia from November 2013 to September 2014 medical care. The study protocol was approved by the Ethical committee of Natural Sciences, Addis Ababa University and confidentiality was assured throughout the study.

\subsection{Clinical Specimens}

Sputum specimens were collected from 248 patients fulfilling the selection criteria. A minimum of 5-7ml morning sputum per patient was collected in sterile, leak-proof, widemouth falcon tube by laboratory professionals in these health institutions and sent to Hawassa Regional Laboratory for storage. The specimens were stored at $+4^{\circ} \mathrm{C}$ and processed within a week.

\subsubsection{Direct Smear Preparation}

Two sets of direct smears were prepared from each sputum sample before concentration by using NACL-NaOH taking a small portion of sputum using applicator stick and evenly distributed smear were made approximately over an area of $1 \times 2 \mathrm{~cm}$ of properly labeled frosted end of microscopic slides. The smears were air dried and heat fixed by passing two to three times over a hot plate. One set of slides were used for $\mathrm{ZN}$ staining and the other set for auramine-O phenol staining method.

\subsubsection{Direct Ziehl-Neelsen Smears Staining}

The prepared smears were placed on the staining rack, heat fixed and stained with $0.3 \%$ carbol fuchsin, heated gently until steam rose, and left for 5 minutes, washed with gentle stream of water and flooded with $3 \%$ acid-alcohol for 3 minutes, washed and flooded with $1 \%$ methylene blue for 1 minute. After one minute methylene blue were washed away, rubbed the back of the slides with dry cotton or gauze, air dried and then observed using 100x oil emersion.

\subsubsection{Fluorochrome Staining}

The prepared slides were placed on a staining rack, heat fixed and stained with auramine-O phenol stain for 20 minutes, then rinsed briefly with gentle stream of water, 
flooded with $0.5 \%$ acid alcohol for 3 minutes then rinsed with water and counter stained with $1 \%$ potassium permanganate for 1 minute [9].

\subsubsection{Sputum Culture}

The sputum specimen was liquefied and decontaminated by N-Acetyl-L-Cysteine Sodium Hydroxide (NALC-NaOH) methods; equal amount of NALC-NaOH solution was added to sputum samples, vortexed and incubated for 15 minutes. After fifteen minutes, the tube filled with sterile distilled water solution up to the top ring on the centrifuge tube (plastic tube has a ring for $50 \mathrm{ml}$ mark), concentrated by centrifugation at a speed of $3,000 \mathrm{xg}$ for 15 minutes and allowed to sit for 5 minutes to settle aerosols. After centrifugation the supernatant was decanted and small quantity (1-2 $\mathrm{ml})$ of distilled water was added and the sediment was re-suspended with the help of a vortex mixer. Three to four drops approximately 100 micro liters of the pellet was inoculated into Lowenstein-Jensen (LJ) media. The media were incubated in slant position for about one week to ensure even distribution of the inoculums. After one week, the tubes were tightened and placed in upright position to minimize evaporation and drying of the media.

\subsubsection{JD-Biotech TB-Antigen Protocol}

Two types of protocols were used to test the sputum sample with JD-TB Ag. One method was with liquefied sputum sample and with viscose sputum samples.

\subsubsection{Protocol for Liquid Sputum Samples}

$500 \mu \mathrm{l}$ of sputum was transfered into the plastic epindroff tube and $500 \mathrm{ml}$ of sample buffer was added, mixed well using vortex mixer, incubated for 30 minutes at $37^{\circ} \mathrm{C}$. After 30 minutes, $200 \mu \mathrm{L}$ of buffer mixed samples was transfered to an eppendorf tube and incubated 60 minutes a $37^{\circ} \mathrm{C}$. Then $100 \mu$ l of sample buffer was added and mixed wel and 4 drops of wel mixed sample was added in the cassette with the plastis pipette and results were red after waiting for 15 minutes.

\subsubsection{Protocol for the Viscose Sputum Samples}

Approximately $500 \mu 1$ of sputum sample was transfered in to the plastic tubes with beads and $1500 \mu \mathrm{L}$ of sample buffer was added and wel mixed with the vortex mixer for two minutes, then incubated for 60 minutes at $37^{\circ} \mathrm{C}$, votexed and incubated for 30 minutes at room temprature, $100 \mu$ l of wel mixed sample was added in the sample pad of the test cassette using plastic dropper and results were red after waiting for 15 minutes.

\subsection{Quality Controls for the Test Procedures}

\subsubsection{Ziehl-Neelsen and Fluorochrome Auramine O-staining Procedures}

Strict procedures were followed during reagent preparation, smear preparation and staining procedures. Known positive and negative sputum specimens were included in every batch of specimens processed. After quality check of the reagent, the study samples were stained using the prepared reagents.
After initial examination, all smears were securely stored in slide boxes. Arbitrary $10 \%$ of the positive smears and $5 \%$ of the negative smears were selected at random and reexamined by Addis Ababa regional laboratory professionals for quality check.

\subsubsection{Quality Control Procedures for Sputum Culture}

Strict procedures were followed from time of media preparation to the time of inoculation. The sterility of the prepared media was checked after 48 hours incubation at $37^{\circ} \mathrm{c}$ to see the aver growth of other contaminants. Inoculation was made in certified bio-safety cabinet level II.

\subsubsection{Safety Issues}

Sputum processing, decontamination, inoculums preparation, inoculation on Lowenstein Jensen media, and slide preparations were performed in a suitable bio-safety level II laboratories dedicated for mycobacterium work with an appropriate ventilation system. Accesses to the room were restricted when work was in progress, and proper protective gowns, gloves and respirator masks (N-95) were used while handling specimens.

\subsection{Data Analysis}

Laboratory results were recorded on excel work sheet during the study period and the collected data from the study area were entered into Microsoft office access and processed using IBM SPSS version 20 statistical software. The sensitivity, specificity, positive and negative predictive values including their $95 \%$ confidence interval (CI) was calculated by using the sputum culture results as the "gold standard". Kappa value was used to determine the statistical agreement of Ziehl-Neelsen stained sputum microscopy, auramine O-stained fluorescence microscopy, JD-TBAntigen detection methods and mycobacterium culture.

\section{Results}

\subsection{Socio-Demographic Characterstics of Study Participants}

In this study, a total of 248 pulmonary tuberculosis presumptive patients were enrolled from November 2013 to September 2014 from different Health Centers and Hospitals in SNNPR of Ethiopia. From the total Presumptive cases, $102(41.5 \%)$ were female. The average age group for the study participants was 35 year and the minimum and maximum age group 18 and 80 respectively.

From 248 study participants screened for pulmonary tuberculosis, $170(68.6 \%)$ were tested for HIV/AIDS of whom 98 (57.65\%) were male and 7 from 98 male screened for HIV, $27(27.55 \%)$ were positive and $71(72.45 \%)$ were negative. Out of 72 female screened for HIV, 27 (37.5\%) were positive.

Treatment history of patients screened for pulmonary tuberculosis were also noted and categorized in to four categories. From the total of 248 pulmonary tuberculosis screened patients, 148 (59.7\%) were new cases, 73 (29.4\%) 
were relapse, $21(8.5 \%)$ treatment failure, $1(0.4 \%)$ defaulter and the remaining $5(2 \%)$ with no recorded treatment history (table 1).

Table 1. Socio-demographic characterstics of study participants by age and sex.

\begin{tabular}{llll}
\hline Age group in years & Male (\%) & Female (\%) & Total (\%) \\
\hline $18-27$ & $39(15.73)$ & $38(15.32)$ & $77(31.05)$ \\
$28-37$ & $57(23)$ & $32(12.90)$ & $89(35.89)$ \\
$38-47$ & $25(10.08)$ & $21(8.5)$ & $46(18.55)$ \\
$48-57$ & $9(3.6)$ & $7(2.8)$ & $16(6.45)$ \\
$58-67$ & $11(4.44)$ & $1(0.4)$ & $12(4.84)$ \\
$\geq 68$ & $5(2)$ & $3(1.2)$ & $8(2.23)$ \\
HIV-status & & & \\
Positive & $27(27.55)$ & $27(37.5)$ & $54(31.76)$ \\
Negative & $71(72.65)$ & $45(62.5)$ & $116(68.24)$ \\
Sex & $145(58.47)$ & $103(41.53)$ & $248(100)$ \\
\hline
\end{tabular}

\subsection{Comparison of ZN Stained Smears and Mycobacterium Culture Results}

From a total of 248 pulmonary tuberculosis suspected patients enrolled in the study; $80(32.23 \%)$ were smear and culture positive, $121(48.79 \%)$ were negative by both methods; 41 (16.53\%) were culture positive but negative by Ziehl-Neelsen stained bright field microscopy. The remaining $6(2.42 \%)$ were positive by $\mathrm{ZN}$-stained bright field microscopy but culture negative (table 2). Based on this study, the SS, SP, PPV and NPV of ZN stained bright field microscopy was $66.12 \%$ (95\% CI: $56.95 \%-74.47 \%), 95.28 \%$ (95\% CI: 89.99\%-98.23\%), 93.02\% (95\% CI: 85.42\%$97.38 \%)$ and $74.69 \% \quad(95 \% \quad$ CI: $\quad 67.27 \quad \%-81.18 \%)$, respectively. The measure of agreement between the two methods were substantial kappa value $(\mathrm{k}=0.618)$.

Table 2. Comparison of ZN Stained Smears and Mycobacterial Culture Results.

\begin{tabular}{llll}
\hline \multirow{2}{*}{ ZN results } & \multicolumn{2}{l}{ Culture Results } & \multirow{2}{*}{ Total (\%) } \\
\cline { 2 - 3 } & Positive (\%) & Negative (\%) & \\
\hline Positive (\%) & $80(32.26)$ & $6(2.42)$ & $86(34.68)$ \\
Negative (\%) & $41(16.52)$ & $121(48.79)$ & $162(65.32)$ \\
Total (\%) & $121(48.79)$ & $127(51.21)$ & $248(100)$ \\
\hline
\end{tabular}

ZN: Ziehl-Neelsen

\subsection{Comparison of Direct Fluorescent Microscopy and Culture Results}

From the total study participants,99 (39.92\%) were positive by both LED-FM and TB culture; 8 (3.23\%) were LED-FM positive but culture negative; 119 (47.98\%) were negative by both methods and the remaining $22(8.87 \%)$ were culture positive but negative by LED-FM microscopy (table 3). The sensitivity, specificity, PPV and NPV of LEDFM were $81.82 \%$ (95\% CI: $73.78 \%-88.24 \%$ ), $93.70 \%(95 \%$ CI: $87.96 \%-97.23 \%), 92.50 \%(95 \%$ CI: $85.79 \%-96.71 \%)$ and $98.35 \%$, respectively. The measure of agreement between the two methods were substantial agreement with kappa value of $(\mathrm{k}=0.757)$.
Table 3. Comparison of Direct Fluorescent Microscopy and Culture results.

\begin{tabular}{llll}
\hline \multirow{2}{*}{ FM results } & \multicolumn{2}{l}{ Mycobacterial culture results } & \multirow{2}{*}{ Total (\%) } \\
\cline { 2 - 3 } & Positive (\%) & Negative (\%) & \\
\hline Positive (\%) & $99(39.92)$ & $8(3.23)$ & $107(43.15)$ \\
Negative (\%) & $22(8.87)$ & $119(47.98)$ & $141(56.85)$ \\
Total (\%) & $121(48.79)$ & $127(51.21)$ & $248(100)$ \\
\hline
\end{tabular}

FM: flourescent microscopy

\subsection{Comparison of LED-FM Microscopy, ZN Microscopy and Culture Method}

Of the total 248 study participants, 99 (39.92\%) were positive by LED-FM and mycobacterial culture but 80 (32.26\%) were positive by $\mathrm{ZN}$ and culture; 121 (48.79\%) were negative by $\mathrm{ZN}$ and mycobacterial culture while 119 (47.98\%) were negative by both LED-FM and culture. Compared to LED-FM, only 6 (2.42\%) of 248 study samples were negative by culture but positive by $\mathrm{ZN}$ where as 8 (3.23\%) samples were positive by LED-FM but negative by mycobacterial culture. On the other hand, 41 (16.53\%) of study particpants were negative by $\mathrm{ZN}$ but positive by culture while $22(8.87 \%)$ were LED-FM negative but positive by culture (table 4).

Table 4. Comparison of LED-FM, ZN stained sputum microscopy and culture results.

\begin{tabular}{llll}
\hline \multirow{2}{*}{ FM } & \multicolumn{2}{l}{ Mycobacterial culture } & Total (\%) \\
\cline { 2 - 4 } & Positive (\%) & Negative (\%) & \\
\hline Positive (\%) & $99(39.92)$ & $8(3.23)$ & $107(43.15)$ \\
Negative (\%) & $22(8.87)$ & $119(47.98)$ & $121(48.79)$ \\
ZN & & & \\
Positive & $80(32.26)$ & $6(2.42)$ & $86(34.68)$ \\
Negative & $41(16.52)$ & $121(48.79)$ & $162(65.32)$ \\
\hline
\end{tabular}

FM: fluorescent microscopy, ZN: Ziehl-Neelsen

\subsection{Comparison of JD-TB-Antigen Test with Culture Method}

A total of 85 specimens were analyzed by using JD-TBAntigen test and culture method. Of the total samples, 21 (24.71\%) were positive by both methods, 15 (17.65\%) were negative by JD-TB-Antigen test but positive by culture, and $18(21.18 \%)$ were culture negative but positive by JD-TBAntigen test. The remaining 31 (36.47\%) were negative by both methods (table 5).

Table 5. Comparison of JD-TB-Antigen and culture results.

\begin{tabular}{llll}
\hline \multirow{2}{*}{ JD-TB-Ag } & \multicolumn{2}{l}{ Mycobacterial culture } & Total (\%) \\
\cline { 2 - 4 } & Positive (\%) & Negative (\%) & \\
\hline Positive (\%) & $21(24.71)$ & $18(21.18)$ & $39(45.88)$ \\
Negative (\%) & $15(17.65)$ & $31(36.47)$ & $46(54.12)$ \\
Total (\%) & $36(42.35)$ & $49(57.65)$ & $85(100)$ \\
\hline
\end{tabular}

The sensitivity, specificity, PPV and NPV of JD-TBAntigen test were $58.33 \%$ (95\% CI: 40.76\%-74.47\%), 63.27\% (95\% CI: $48.29 \%-76.57 \%), 53.85 \%$ at (95\% CI: $37.19 \%-69.90 \%)$ and $67.39 \%$ (95\% CI: $51.98 \%-80.46 \%$ ), respectively. The two methods have shown slight agreement with kappa value of (0.046) (fig 1 and 2). 


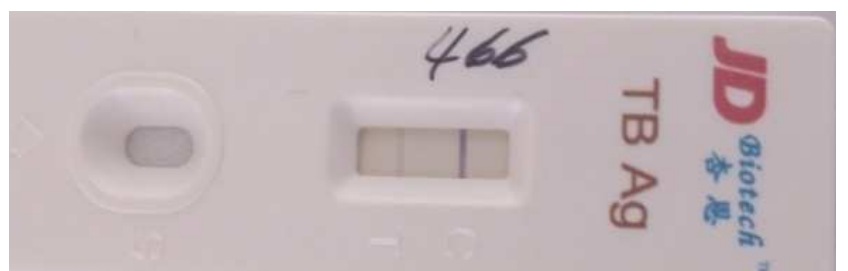

Figure 1. JD-TB-Antigen positive results.

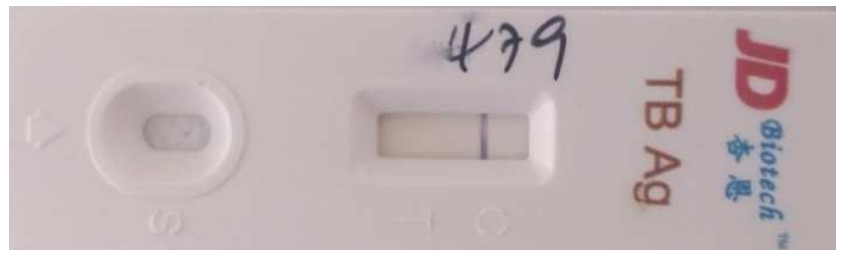

Figure 2. JD-TB Antigen negative results.

\section{Discussion}

In the present study, we compared the performances of light-emitting diode fluorescent microscopic (LED-FM), Ziehl-Neelsen stained sputum microscopy and JD-TBAntigen test with that of TB culture as a reference. Our study result showed that the sensitivity of $\mathrm{ZN}$ stained bright field microscopy was $66.12 \%$ which is similar to the findings (sensitivity of $66 \%$ ) reported from Nairobi but higher than the results (sensitivities $(62.3 \%$ and $64 \%)$ reported from Uganda. The difference might be associated with the status of the study participants. The study done in Uganda was carried out on HIV/AIDS patients but in our cases the study was conducted regardless of HIV status of the patients'. The findings reported by Bonnet and his colleagues showed higher sensitivity (72\%) than the present result. This difference might be attributed to the difference in number of sample size. Bonnet and his collogues used almost twice our sample size $[10,11]$.

In our study, the specificity $\mathrm{ZN}$ stained microscopy is $95.28 \%$ which is almost similar to the findings reported from Bangladesh specificity (97.61\%) and Ethiopia (specificity of $(96.5 \%))([12,13]$. When this finding is compared to the previous study results $(91.3 \%)$ reported by Rahman and his colleagues, the specificity is higher [14]. The study in Bangladesh was done on both patients taking antituberculosis and newly diagnosed patients which lower sensitivity and specificity of the test methods.

In our study, the sensitivity of light-emitting diode fluorescent microscopy was $81.82 \%$ which is lower than sensitivity (84.7\%) reported from South Africa, Cape Town [6]. The study in South Africa was conducted by bleach concentration which might increases bacillary load to be detected and increase sensitivity of the test method.

As compared to $\mathrm{ZN}$ stained bright field microscopy (sensitivity $66.12 \%$ ), the sensitivity of LED-FM was $81.82 \%$, with incremental yields of $15.7 \%$. However, the specificity of LED-FM has $93.70 \%$ shown lower specificity (95.28\%) compared to $\mathrm{ZN}$ stained sputum microscopy. Reports of WHO, 2010 indicated 10\% incremental yields in sensitivity of LED-FM with comparable specificity of ZN stained bright field microscopy [19].

In this study, JD-TB-Antigen detection method has shown the sensitivity of $58.33 \%$ which is lower than sensitivity (69.0\%) reported from Southern Italy ([17]. Reports of WHO, 2010 on the Pathozyme Myco, IgG, IgM and IgA indicated the sensitivity ranging from $10 \%-85$. Findings from Thailand indicated sensitivity of $48 \%$ which is lower than the sensitivity (58.33\%) of our finding. But the study in Thailand was done on HIV/AIDS patients whom may vary in immune response characteristics and the target gene immunochromatographic test might different. Specificity (63.27\%) of our study is much lower than the findings of Alifano and his colleagues (specificity of $93.0 \%$ ) but higher than reports of Nanta and colleagues finding specificity $52 \%$ [18]. Lee and his colleagues reported specificity of (96.7\%) [19] which is higher than our finding. But their study was done on Mycobacterium culture positive samples which probably increased the sensitivity and specificity of the test method.

\section{Conclusion and Recommendation}

Based on the findings of this study, the following conclusion was drawn. LED -FM has shown better sensitivity $(81.82 \%)$ in diagnosis of pulmonary tuberculosis compared to the sensitivity $(66.12 \%)$ of Ziehl-Neelsen stained bright field microscopy. The specificities of ZiehlNeelsen (95.23\%) and light-emitting diode fluorescent microscopy $(93.70 \%)$ were almost equivalent. LED based fluorescent microscopy is more sensitive, easy to perform, inexpensive and decrease time taken for microscopic examination of pulmonary tuberculosis. This is especially useful in developing countries where resources are limited for utilization of culture and molecular techniques. The sensitivity and specificity of JD-TB-Antigen test was found to be low. Therefore, it is better to use LED-FM for the diagnosis of pulmonary tuberculosis as an alternative diagnostic method to $\mathrm{ZN}$ stained sputum microscopy. Large scale study should be done for evaluation of the performance of JD-TB-Antigen test to be used as the diagnostic method for pulmonary tuberculosis in our setting.

\section{Annexes}

A1.

Ziehl-Neelsen Staining Reagents Preparations

CarbolFuchsin (3\%) Quantity per liter

Solution 1

Basic fuchsin: $3.0 \mathrm{~g}$

Denatured alcohol or methanol (95\% ethanol): $100.0 \mathrm{ml}$

Dissolve basic fuchsin in ethanol

Phenol

Phenol crystals (technical grade): $50 \mathrm{~g}$

Distilled water (purified water): $850 \mathrm{ml}$

Dissolve phenol crystals in distilled water (gentle heat may

be required) Solution 2

Working solution 
Combine $10 \mathrm{ml}$ of solution 1 with $90 \mathrm{ml}$ of solution 2 and store in an amber bottle. Label bottle with name of reagent as well as preparation and expiry dates. Store at room temperature for six to twelve months and filter before use

Decolorizing agent: $3 \%$ acid-alcohol

Concentrated hydrochloric acid (technical grade): $30 \mathrm{ml}$

Alcohol, 95\% ethanol: $970 \mathrm{ml}$

Carefullyadd concentrated hydrochloric acid to $95 \%$ ethanol. Store the reagent in amber bottle and label bottle with name of reagent and date of preparation and expiry date. Store at room temperature for six to twelve months

Counterstain: Methylene blue (0.3\%)

Methylene blue chloride: $3.0 \mathrm{~g}$

Distilled water: $1000.0 \mathrm{ml}$

Dissolve methylene blue chloride in distilled water and store in an amber bottle. Label bottle with name of reagent and dates of preparation and expiry. Store at room temperature for six to twelve month

A2.

Fluorochrome Staining Reagent Preparation

Auramine O (0.1\%)

Auramine: $1.0 \mathrm{~g}$

95\% ethanol (technical grade): $100 \mathrm{ml}$

Dissolve auramine in ethanol Solution 1

Phenol

Phenol crystals (analytical grade): $30.0 \mathrm{~g}$

Distilled water: $870 \mathrm{ml}$

Dissolve phenol crystals in water Solution 2

Mix solutions 1 and 2 and store in a tightly stoppered amber bottle away from heat and light. Label bottle with the name of the reagent and dates of preparation and expiry date. Stored at room temperature for three months. Turbidity may develop on standing but this does not affect the staining reaction.

Decolorizing solution

Concentrated hydrochloric acid (37\%): $5 \mathrm{ml}$

Denatured 95\% ethanol (technical grade): $995 \mathrm{ml}$

Carefully add concentrated hydrochloric acid to the ethanol. Store in an amber bottle and labeled the bottle with name of reagent and dates of preparation and expiry. Store at room temperature for three months

Counter stains

Potassium permanganate: $(0.5 \%)$

Potassium permanganate $(\mathrm{KMnO} 4)$ certified grade: $5.0 \mathrm{~g}$

Distilled water: $1000 \mathrm{ml}$
Dissolve potassium permanganate in distilled water in a tightly stoppered amber bottle. Label bottle with name of reagent and dates of preparation and expiry. It can be stored at room temperature for about three months.

Table A1. Preparation of NALC-NaOH Digestant-Decontaminant Solution.

\begin{tabular}{llll}
\hline $\begin{array}{l}\text { Volume of } \\
\text { digestant In } \\
\text { needed }(\mathbf{m l})\end{array}$ & \multicolumn{2}{c}{ Mix indicated amount $(\mathbf{m l})$ of } & Add NALC \\
\cline { 2 - 3 } & $\mathbf{4 \%} \mathbf{N a O H}(*)$ & $\begin{array}{l}\mathbf{2 . 9 \%} \text { Na citrate } \\
\mathbf{2 H 2 O}(+)\end{array}$ & \\
\hline 50 & 25 & 25 & 0.25 \\
100 & 50 & 50 & 0.50 \\
200 & 100 & 100 & 1.00 \\
500 & 250 & 250 & 2.50 \\
1000 & 500 & 500 & 5.00 \\
\hline
\end{tabular}

(*) Add $4.0 \mathrm{~g} \mathrm{NaOH}$ to $100 \mathrm{ml}$ distilled water

(+) Add $2.9 \mathrm{~g}$ sodium citrate dihydrate (or $2.6 \mathrm{~g}$ anhydrous sodium citrate) to $100 \mathrm{ml}$ of distilled water

Table A2. Preparation of Modified Lowenstein-Jensen.

\begin{tabular}{|c|c|c|}
\hline S. $\mathbf{N}$ & Mineral contents & amounts \\
\hline 1 & MonoPotacium phosphate (anhydrous) & $2.4 \mathrm{~g}$ \\
\hline 2 & Magnesium Sulphate (MgSO4. 7H2O) & $0.24 \mathrm{~g}$ \\
\hline 3 & Magnesium Citrate & $0.6 \mathrm{~g}$ \\
\hline 4 & Asparagine & $3.6 \mathrm{~g}$ \\
\hline 5 & Glycerol (reagent grade) & $12.0 \mathrm{ml}$ \\
\hline 6 & Distilled water & $600.0 \mathrm{ml}$ \\
\hline 7 & Autoclave at $121^{\circ} \mathrm{c}$ for 30 minutes & \\
\hline 8 & Cool to room temperature & \\
\hline 9 & $\begin{array}{l}\text { Add malachite green ( } 2 \% \text { aqueous solution, freshly } \\
\text { prepared) }\end{array}$ & $20.0 \mathrm{ml}$ \\
\hline 10 & Add homoginized whole eggs & $1000.0 \mathrm{ml}$ \\
\hline 11 & $\begin{array}{l}\text { Mix and pour into a sterile aspirator bottle or funnel } \\
\text { tubes (test tube device) and dispense }\end{array}$ & \\
\hline 12 & $\begin{array}{l}\text { Place approximatley6-8ml into each } 20-150 \mathrm{~mm} \\
\text { sterile screw cup tube }\end{array}$ & \\
\hline 13 & $\begin{array}{l}\text { Slant and coagulate by inspassation at } 85^{\circ} \mathrm{c} \text { for } 50 \\
\text { minutes. Incubate at } 37^{\circ} \mathrm{c} \text { for } 48 \text { hoursas sterility } \\
\text { check. Medium may be storedin refrigerator for } \\
\text { several months and if caps are tightly closed to } \\
\text { prevent evaporation }\end{array}$ & \\
\hline
\end{tabular}

Note-Fresh hens' eggs, not more than seven days old, are cleaned by scrubbing thoroughly with a hand brush in warm water and a plain alkaline soap. Let the eggs soak for 30 minutes in the soap solution. Rinse eggs thoroughly in running water and soak them in $70 \%$ ethanol for 15 minutes. Before handling the clean dry eggs scrub the hands and wash them. Crack the eggs with a sterile knife into a sterile flask and beat them with a sterile egg whisk or in a sterile blender.

Table A3. IUATLD/WHO recommended grading of sputum microscopy results.

\begin{tabular}{|c|c|c|c|}
\hline \multirow[b]{2}{*}{$\begin{array}{l}\text { IUATLD/WHO scale } \\
(1000 x \text { fields })=\text { HPF } \\
\text { Results }\end{array}$} & \multicolumn{3}{|l|}{ MICROSCOPY SYSTEM USED } \\
\hline & $\begin{array}{l}\text { Bright field }(1000 \times \text { Magnification })=1 \text { length }=2 \\
\mathrm{~cm}=100 \mathrm{HPF}\end{array}$ & $\begin{array}{l}\text { Conventional fluorescence }(200-250 x \\
\text { magnification })=1 \text { length }=30 \\
\text { fields }=300 \mathrm{HPF}\end{array}$ & $\begin{array}{l}\text { iLED fluorescence } \\
\text { (400xmagnification; } 1 \\
\text { length }=40 \text { fields }=\mathbf{2 0 0} \mathrm{HPF}\end{array}$ \\
\hline Negative & Zero AFB/1 length & Zero AFB/1 length & Zero AFB/1 length \\
\hline Scanty (actual count) & 1-9AFB/1 lengthor100 HPF & 1-29AFB/1 length & 1-19 AFB/1 length \\
\hline $1+$ & 10-99 AFB/1 lengthor100 HPF (1-9AFB/10field & 30-299 AFB/1 length & 20-199 AFB/1 length \\
\hline $2+$ & 1-10 AFB/1 HPF on average & $10-100 \mathrm{AFB} / 1$ Field on average & 5-50 AFB/1 Field on average \\
\hline $3+$ & $>10 \mathrm{AFB} / 1$ Field on average & $>100 \mathrm{AFB} / 1$ Field on average & $>50 \mathrm{AFB} / 1$ Field on Average \\
\hline
\end{tabular}




\section{References}

[1] FMoH. guide lines for clinical and programmatic managements of TB, TB/HIV and Leprosy in Ethiopia $5^{\text {th }}$ edn 2013.

[2] Parsons, L. M., Somoskövi, A., Gutierrez, C., Lee, E., Paramasivan, C. N., Abimiku, A., etal. Laboratory diagnosis of tuberculosis in resource-poor countries: challenges and opportunities. Clin. Microbiol. Rev. 2010: 24: 314-345.

[3] FMoH. National HIV/AIDS Prevention Control Office Technical Document for the AIDS in Ethiopia $6^{\text {th }}$ Report. 2009.

[4] WHO. Global tuberculosis report. Geneva, Switzerland. 2013.

[5] Mathur, M. L., Gaur J., Sharma R. and Solanki, A. Rapid culture of Mycobacterium tuberculosis on blood agar in resource limited setting. Dan Med Bull. 2009: 56: 208-210.

[6] Marais, B. J., Brittle, W. and Painczyk, K. Use of lightemitting diode fluorescence microscopy to detect acid-fast bacilli in sputum. Clin Infect Dis. 2008: 47: 203-207.

[7] Steingart, K. R., and Henry, M. N. V. Fluorescence versus conventional sputum smear-microscopy for tuberculosis: a systematic review. Lancet Infect Dis. 2006: 6: 570-581.

[8] Van Deun, A., Chonde, T. M., Gumusboga, M., Rienthong, S. Performance and acceptability of the Fluo LED Easy module for tuberculosis fluorescence microscopy. Int $J$ Tuberc Lung Dis. 2008: 12: 1009-1014.6.

[9] WHO. Laboratory services in tuberculosis control. Geneva, Switzerland, 1998.

[10] Albert, H., Manabe, Y., Lukyamuzi, G., Ademun, H, Mukkada, S. Nyesiga, B., Joloba, M., Paramasivan, C. N. and Perkins, M. D. Performance of Three LED-Based Fluorescence Microscopy Systems for Detection of Tuberculosis in Uganda. PLoS ONE 2010: 5: 1-7.

[11] Bonnet, M., Gagnidze, L., Githui, W., Guerin,. P. J., Bonte, L.,
Varaine.F.e tal. A. Performance of LED-Based Fluorescence Microscopy to Diagnose Tuberculosis in a Peripheral Health Centre in Nairobi. PLoS ONE. 2011: 6: 1-6.

[12] Khatun, Z., Hossain, M. S., Roy, C. K., Sultana, T., Rahman, Azad, S. e tal. Light Emitting Diode (LED) Fluorescent Microscopy: a Milestone in the Detection of Paucibacillary Mycobacterium in Case of Pulmonary Tuberculosis. Bangladesh Medical Journal. 2011: 40: 1-22-26.

[13] Cuevas, L. E., Al-Sonboli, N., Lawson, L., Yassin, M. A., Arbide, I., Al-Aghbari, N., et al. A. LED Fluorescence Microscopy for the Diagnosis of Pulmonary Tuberculosis: A Multi-Country Cross-Sectional Evaluation. 2011: 8: 1-10.

[14] Rahman, F., Munshi, S. K., Mostofa Kamal S. M., MatiurRahman, S. M., Rahman, M. M. and Noor, R. Comparison of Different Microscopic Methods with Conventional TB Culture. S. J. Microbiol. 2011: 1: 20745356.

[15] Cattamachi, A., Davis, J. L., Worodria, W., Boon, S., Yoo, S., Matovu, J., et al. Sensitivity and Specificity of Fluorescence Microscopy for Diagnosing Pulmonary Tuberculosis in a High HIV Prevalence Setting. Int J Tuberc Lung Dis. 2009: 13: $1130-1136$.

[16] WHO. Commercial Sero diagnostic Tests for Diagnosis of Tuberculosis. 2010.

[17] Alifano, M., Pascalis, R. D., Sofia, M., Faraone, S., Pezzo, M., and Covelli, I. Detection of $\operatorname{IgG}$ and $\operatorname{IgA}$ against the mycobacterial antigen A-60 in patients with extrapulmonary tuberculosis. Thorax. 1998: 53: 377-380.

[18] Nanta, S., Kantipong, P., Pathipvanich, P., Ruengorn, C., Tawichasri, C. and Patumanond, J. Diagnostic value of an immunochromatographic test over clinical predictors for tuberculosis in HIV patients. Clinical Epidemiology. 2011: 3: 237-244.

[19] Lee, J., Yu, F., Lin, M., Huang, G., Chang, C., Cheng, C., and Wang. G. Utility of Immunochromatographic Assay for Detecting Mycobacterium Tuberculosis from Positive BACTEC MGIT 960 Cultures. J Biomed Lab Sci. 2010: 22: 64-68. 